
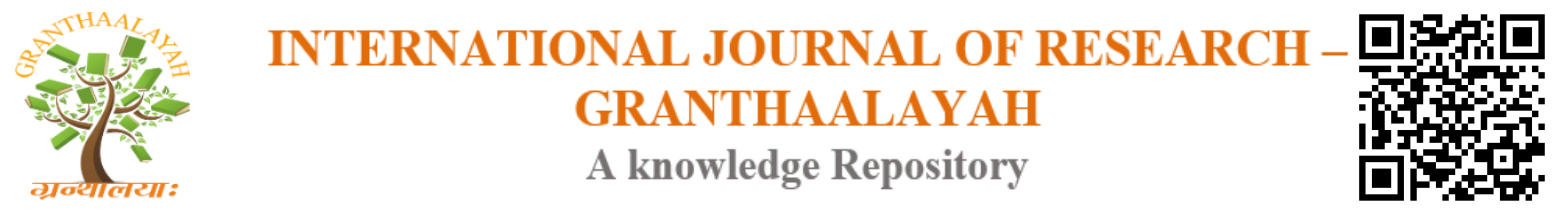

Science

\title{
INTRODUCING IN VITRO EXPERIMENTS OF OXYGEN BUBBLES SHOCKWAVES TRIGGERING INTRACELLULAR LIPIDS LUMINESCENCE: IMPLICATIONS IN CANCER ETIOLOGY
}

\author{
Abraham A. Embi Bs MBA *1 \\ ${ }^{* 1} 13442$ SW 102 Lane, Miami, Florida USA, 33186
}

\begin{abstract}
Background

The main purpose of this manuscript is to introduce a mechanism supporting a previously hypothesized factor in cancer origin, where endogenous energy emission during cell respiration was identified as additional factor in cancer origin. Recent published reports identify the pressure profile of shockwaves as causing lipid droplets membrane deformation. Lipid metabolism has been highlighted to have a key role in cancer metabolism, and metastasis; for example, several publications have suggested targeting lipid metabolism of cancer cells as a strategy to control metastasis. New studies have revealed that lipid layers are responsible for the storage and discharge of static electricity. This manuscript introduces shockwaves from oxygen bubbles bursts as a mechanism causing intracellular lipids discharge or static electricity. The effect causes shape changes of lipid droplets up to a light emission stage.

\section{Materials and Methods}

Cheek cells intracellular material, including DNA strands and lipid droplets were precipitated in a test tube by following written instructions on DNA precipitation published online by The University of Michigan. The DNA precipitate was transferred onto a clean glass slide and covered by a similar one and dubbed a sandwich (SDW). A slide assembly was developed where the effect of oxygen bubbles cavitation-induced shockwaves on the trapped DNA precipitate and lipid droplets were recorded. Microphotographs and video recordings were stored in a computer via a video-microscope.
\end{abstract}

\section{Results}

Lipid droplets exposed to prolonged shockwaves energy were documented to undergo recurrent expanding architectural deformation up to a final contracting phase where light was emitted.

\section{Conclusions}

Intracellular lipid droplets are ubiquitously present in cells; and recent research has shown their expanded roles in cellular signaling in both mitotic and non-mitotic cells. In cancer, one highlighted key role is the potential of lipid metabolism in metastatic colonization. Data introduced in this manuscript demonstrates a direct consequence of $\mathrm{ROS}\left(\mathrm{H}_{2} \mathrm{O}_{2}\right)$ decomposition (via oxygen bubbles bursts) as a trigger for lipid intracellular droplets emission of light radiation, thus supporting a previously proposed biophysical mechanism in cancer origin. 


\section{Implications}

The introduced energy accumulation and discharge by the lipid droplets could be inferred as to alter the lipid molecules intracellular functions, with probable implications in diseases, including cancer genesis. Further research is warranted.

Keywords: Intracellular Lipids Membrane; Cancer Genesis; Lipid Droplets Luminescence; DNA Damage; $\mathrm{H}_{2} \mathrm{O}_{2}$ Breakdown; Bursting Oxygen Bubbles; Cancer Immortality; DNA Lipid Bonding.

Cite This Article: Abraham A. Embi Bs MBA. (2019). "INTRODUCING IN VITRO EXPERIMENTS OF OXYGEN BUBBLES SHOCKWAVES TRIGGERING INTRACELLULAR LIPIDS LUMINESCENCE: IMPLICATIONS IN CANCER ETIOLOGY." International Journal of Research - Granthaalayah, 7(4), 355-364. https://doi.org/10.29121/granthaalayah.v7.i4.2019.921.

\section{Introduction}

The main objective in this manuscript is the introduction of the noticeable effect of bursting oxygen molecules on intracellular lipid droplets leading into radiation emitted in the form of light. This phenomenon falls in the category of a static electricity discharge where heat was not a trigger. Endogenous irradiation in the living cells "could lead to cancer" (1), this theory was originally proposed in 1956, and expanded in 2016 by the hypothesizing that electromagnetic forces during cell respiration are an additional factor in disease genesis, including cancer (2). In the medical literature the emphasis on the cancer war has been mainly concentrated in attributing intracellular molecular signaling to cancer genesis, metastasis and possible cure $(3,4,5)$. In a recent publication, "the static electricity of regenerative natural substances has been a long-term curiosity in human history, it is not yet clear which of their components causes the positive static charges. This study reveals that lipid layers on the surface of regenerative substances (skin, hair, leaves, cells) and even synthetic lipids are responsible" (6). This manuscript further expands the concept of ROS generated energy as factor in cancer origin. How? By introducing in vitro data showing energized intracellular lipid droplets caused by energy emitted from bursting of oxygen bubbles penetrating a thin glass barrier. During homeostasis ROS, (such as the $\mathrm{H}_{2} \mathrm{O}_{2}$ molecule), is broken down generating water and oxygen molecules that combine to others producing oxygen bubbles. These bubbles eventually burst and emit shockwaves known to induce prokaryotic and eukaryotic cells deformations (7). The architectural shape changes (of the lipid droplets) could also be theorized to induce cellular metabolic changes of unknown consequences; but is that enough to explain cancer genesis? In this manuscript, additional effects of the shockwaves in causing lipid droplets architectural changes up to emitting light radiation a.k.a. as luminescence (Figure 1), (Figure 2) (plus video embedded in Figures) are introduced. This finding could lead into a new paradigm in cancer origin and pathogenesis, such as biophysical phenomena inducing molecular changes. 

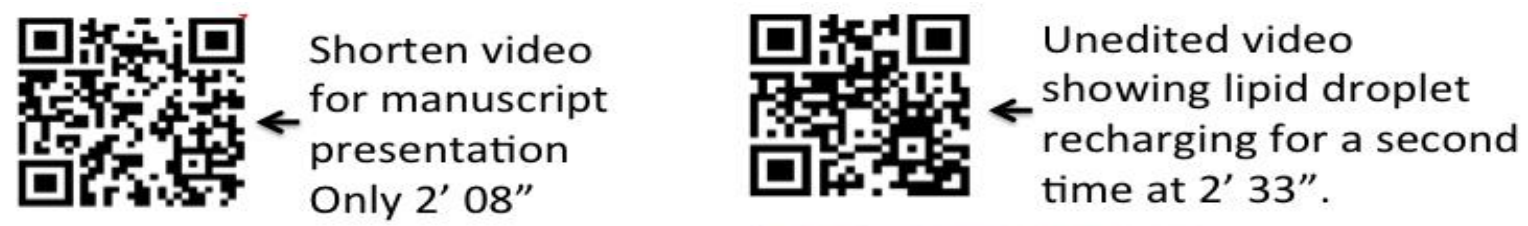

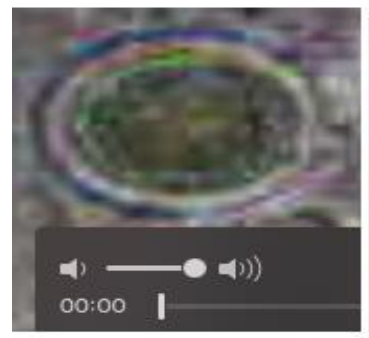

RECORDING BEGINS

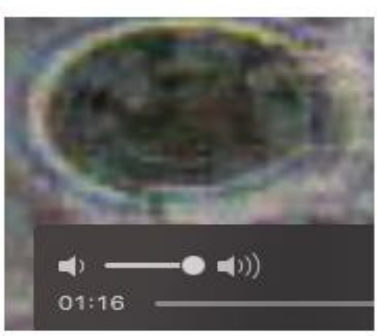

$1^{\prime} 16^{\prime \prime}$

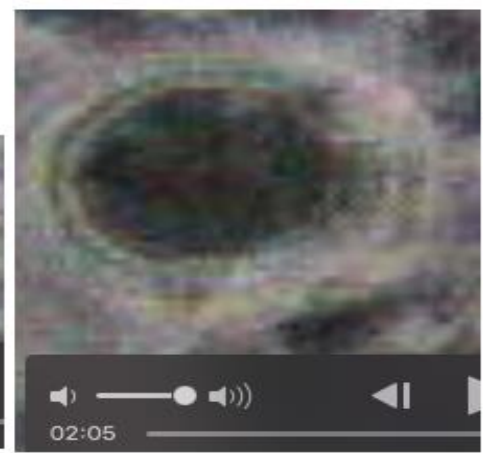

2'06”

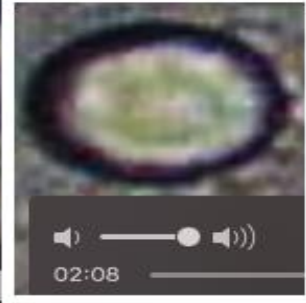

2'08”

Figure 1: Serial video frames of a lipid droplet undergoing expansion and contraction up to exhibiting luminescence. Notice $\mathrm{O} 2$ bubbles bursting in background. This phenomenon triggered by repeated $\mathrm{O}_{2}$ bubbles bursting and releasing energy penetrating through a $0.017 \mathrm{~mm}$ thick glass barrier. For additional details link to: https://youtu.be/lWD5sGPiUpU

Or Scan the QR Code on image with smartphone.
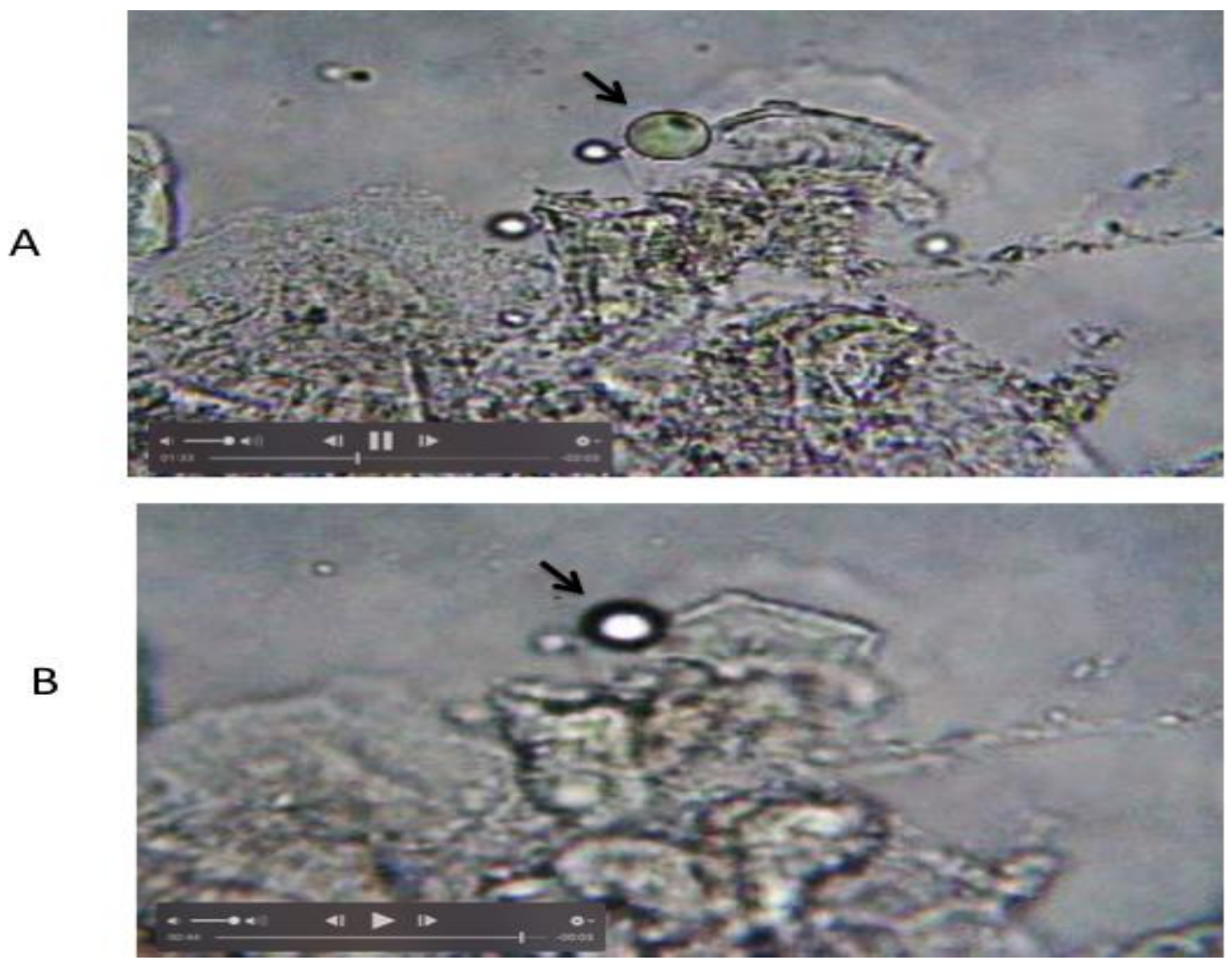

Figure 2: Additional demonstration of lipid droplet luminescence. 
Panel $\mathrm{A}=$ Black arrow pointing at lipid droplet control.

Panel $\mathrm{B}=$ Showing lipid droplet luminescence. Notice the sudden burst of light causing the image to be out of focus. Notice in panel A smaller lipid droplets that were already in a luminescent phase.

For additional details, please link to: https://youtu.be/pVYQD64PB-o

\section{Materials and Methods}

Written online instructions by The University of Michigan on how to harvest DNA fibers were followed and DNA white clumps are seen and harvested from a test tube (8). A slide assembly was constructed where on a $25 \times 75 \times 1 \mathrm{~mm}$ clean glass slide; fragments of thin sliced processed meat (salami) were placed. The fragments were separated by approximately a $5 \mathrm{~mm}$ gap. One small drop of $35 \% \mathrm{H}_{2} \mathrm{O}_{2}$ was placed on each fragment, oxygen bubbles ensued. Previously, drops of precipitated DNA white clumps were harvested by a wooden stick and sandwiched (SDW) between two $25 \times 50 \times 0.017 \mathrm{~mm}$ cover slides. The SDW was carefully centered by visually aligning the center with the $5 \mathrm{~mm}$ gap between the salami fragments. Microphotograph and video recordings were done via a video microscope (Celestron Model \# 44348) and downloaded into an Apple computer Photo App for further evaluation.

\section{Results}

\section{Static Electricity from Shockwaves}

Focusing on a lipid droplet, continuous video recordings were obtained $(n=5)$ while the area was affected by shockwaves from during oxygen bubbles bursting. Gradual changes in shape, such as expansion/contraction of the lipid, as well as a circular aura was observed. Period A sudden burst in brightness (light) from the droplet caused the image to be out of focus. The video recording (Figure 1 above) clearly shows motion of the lipid molecules and clumping DNA fibers caused by the oxygen bubbles shockwaves seen in the background.

\section{Discussion}

During the DNA harvest procedure cells membranes are purposely broken by surfactants, the intracellular material spills into the test tube; and the addition of a protease reduces the amount of protein when the DNA is precipitated. Mixed with the DNA clumps were intracellular lipid droplets of different sizes, which were identified as such due to their malleability and failure to coalesce with drifting oxygen bubbles (Figure 3, Figure 4). As a note of interest, lipid droplets malleability had also been confirmed in cancer cells. 


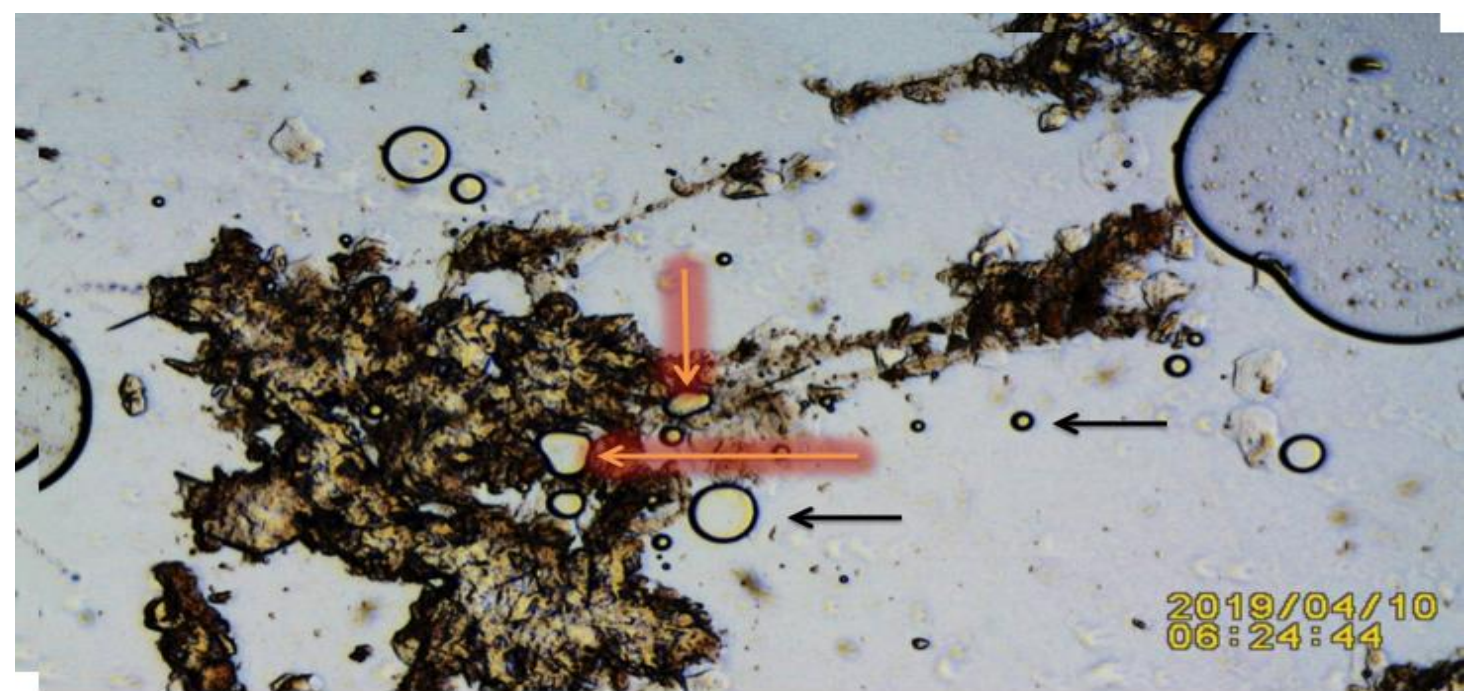

Figure 3: Microscopy image (X20 Magnification) of DNA precipitate and lipid droplets. Notice

material clump from white sticky DNA fibers precipitate. Black Arrows= Different sizes intracellular lipid droplets. Orange Arrows= Pointing at deformed lipid droplets trapped within the precipitate.

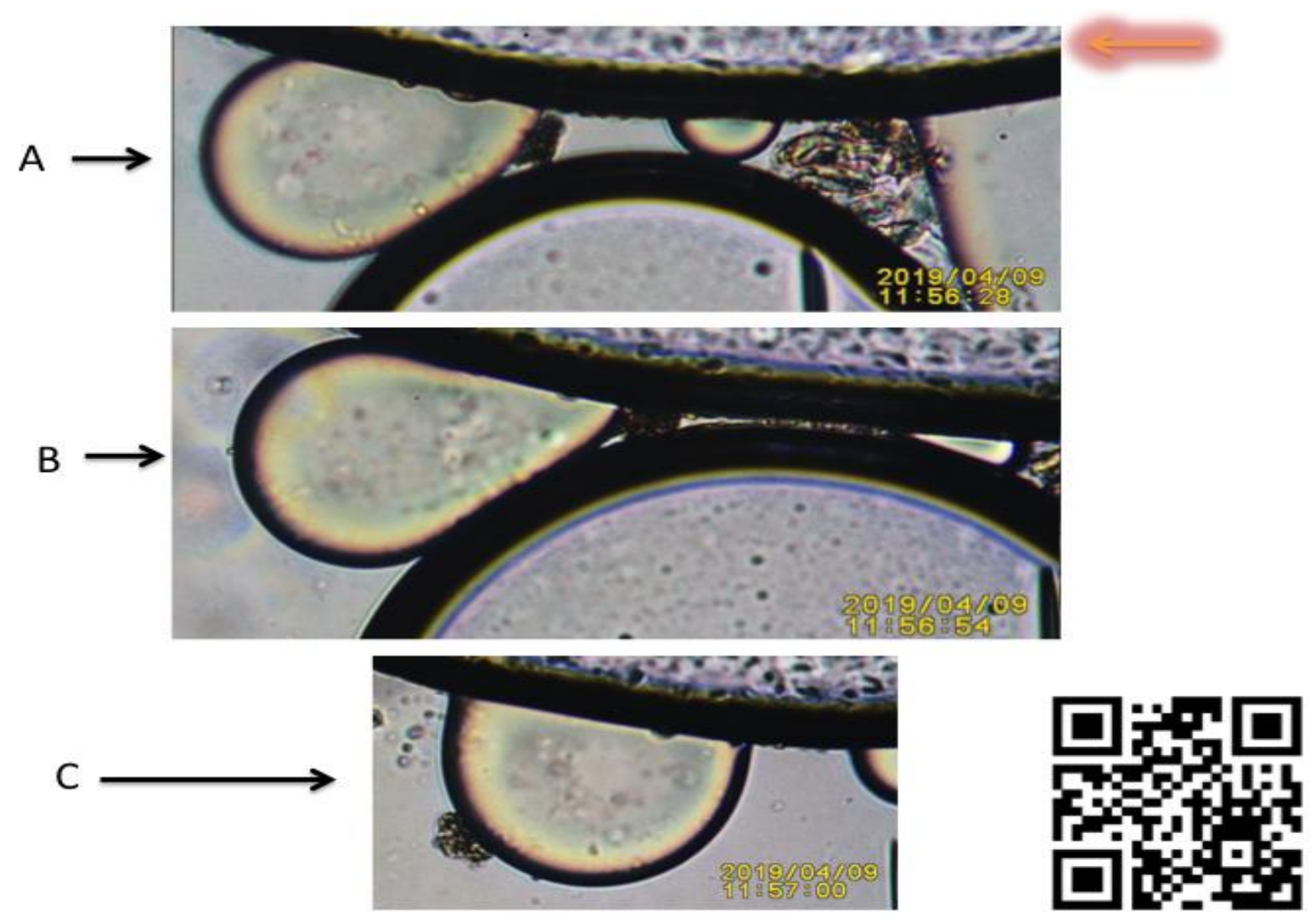

Figure 4: The malleability of intracellular lipid cells not merging with oxygen bubbles. Upper Right Highlighted Orange Arrow= Oxygen bubble. A,B= Notice the temporary deformations resembling teardrop. $\mathrm{C}=$ Once the bubble's mechanical pressure ceases, lipid droplet reverts to a rounded shape.

For additional information link to: https://youtu.be/_liuE8vfXVs Or scan QR Code in image. 


\section{Present Paradigm in Cancerology}

\section{The Molecular Approach}

Lipids have been suggested to fuel cancer (9); the cancer lipidome is "diverse and malleable, with the ability to promote or inhibit cancer pathogenesis...targeting lipids within the tumor and surrounding microenvironment may be a novel therapeutic approach" (10); again researches are recommending a molecular approach in the cancer war.

\section{Keratinocytes Lipids and Cancer}

Numerous papers are also linking keratin, ROS and lipids to cancer $(11,12)$. Of relevancy to the findings in this manuscript is the prevalence of lipid stores in keratinocytes in human skin (13). This researcher is recommending a combined Biophysical/Molecular approach in the cancer war, since there is a possibility that by blocking bursting oxygen bubbles shockwaves could reduce the lipid molecule luminescence and consequences. There are other published hypotheses supporting this concept $(14,15)$

\section{The Rechargeable Lipid Droplet as Energy}

As a final note in this complex matter, the video recordings QR Codes imbedded in the figures, confirm a property of a lipid droplet, namely a regenerative capacity such as the storing and releasing of energy as long as the shockwaves causing the unstable environment persist.

\section{Conclusions}

It is known that shockwaves arising from ROS breakdown create oxygen bubbles. That these bubbles rupture and emit energy in the form of shockwaves. That when a surge of electrons reach a lipid droplet energy transfer ensues. Presented herein is the luminescence phenomenon seen in lipid droplets once an energy saturation point is achieved. Lipids are categorized as a "regenerative substance" (16), this property is confirmed by data demonstrating the recurrent discharging of energy by lipids as long as the endogenous energy (shockwaves) persists (Figure 5, Figure 6, Figure 7, Figure 8). Proposed is that the luminescence occurs to maintain the intracellular space in equilibrium, thus possibly causing damage to surrounding structures including DNA. The biophysical mechanism described above is "a fit" in explaining DNA damage resulting from ROS decompositions. After all DNA has been reported to bind to lipid droplets (17). 


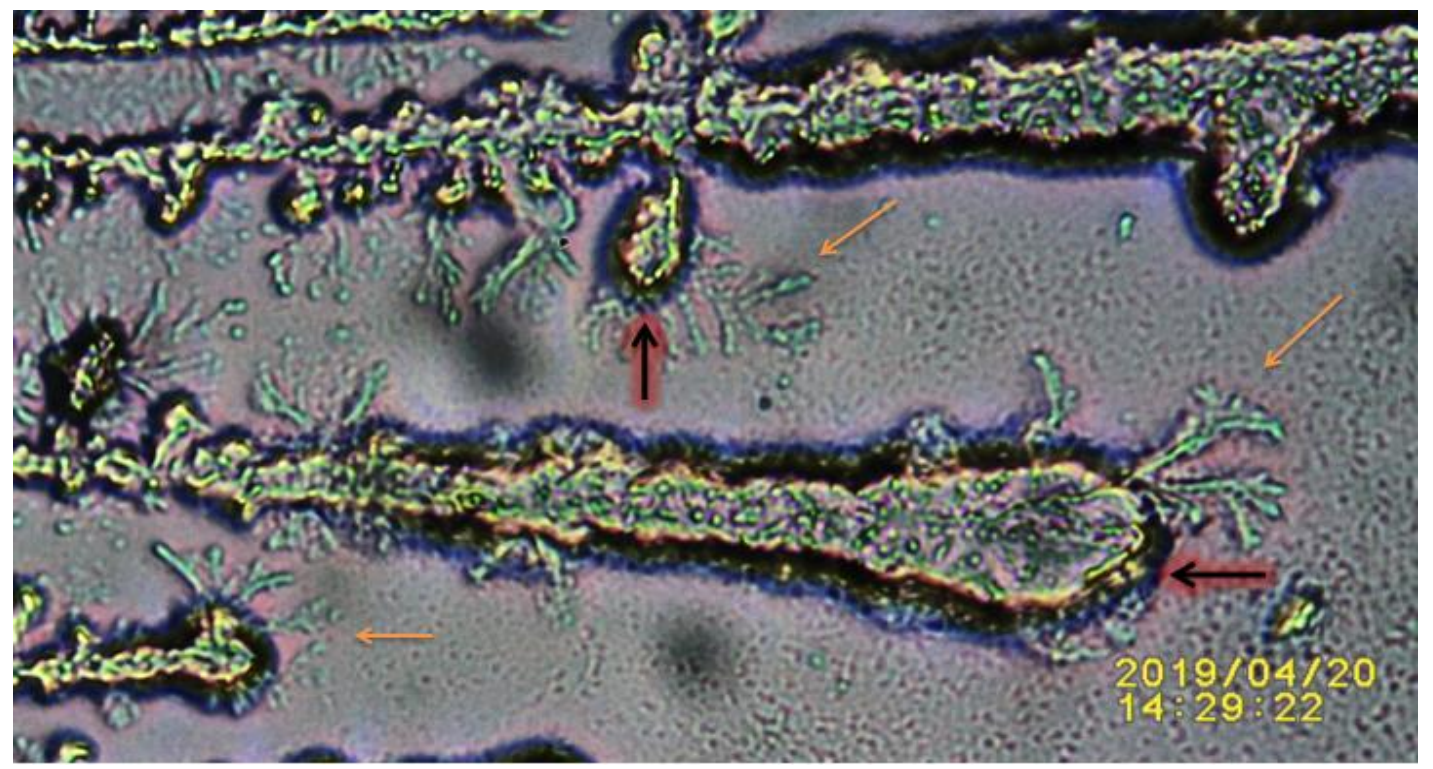

Figure 5: Amplified saliva crystals after $\mathrm{O}_{2}$ bubbles cavitation shockwaves stimulation. Orange Arrows $=$ Pointing at electrical discharges. Black Arrows $=$ Pointing at distal ends of crystallized saliva.

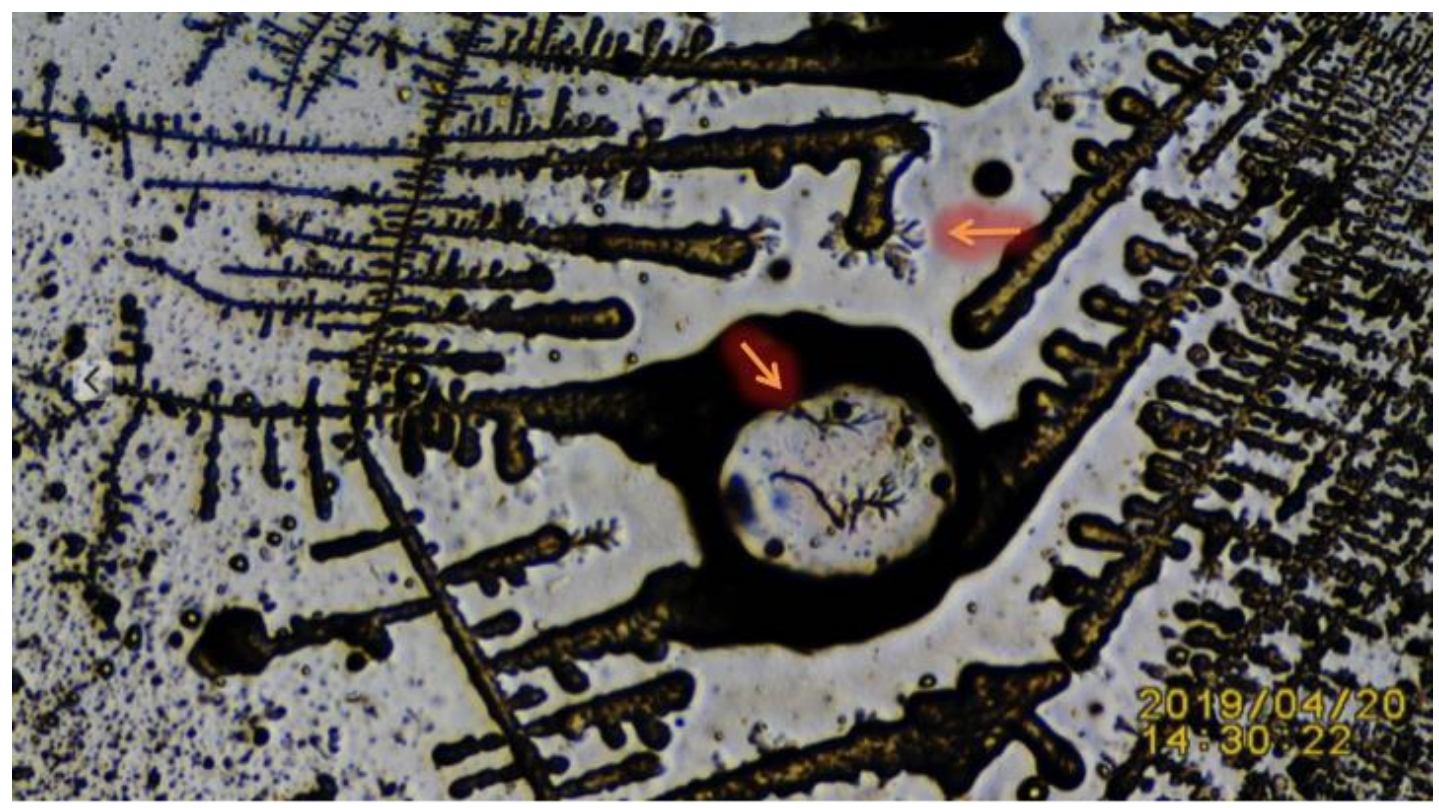

Figure 6: Demonstration of O2 Bubble optically amplifying static electricity discharge (into O2 bubble) from dry saliva crystals. Saliva drops on SSP cover slide- Salami $1 \mathrm{~mm}$ SDW $+35 \%$ $\mathrm{H} 2 \mathrm{O} 2$. Highlighted Orange Arrows= Highlighting saliva crystal electrical discharges, inside and outside air bubble. X10 Magnification. 


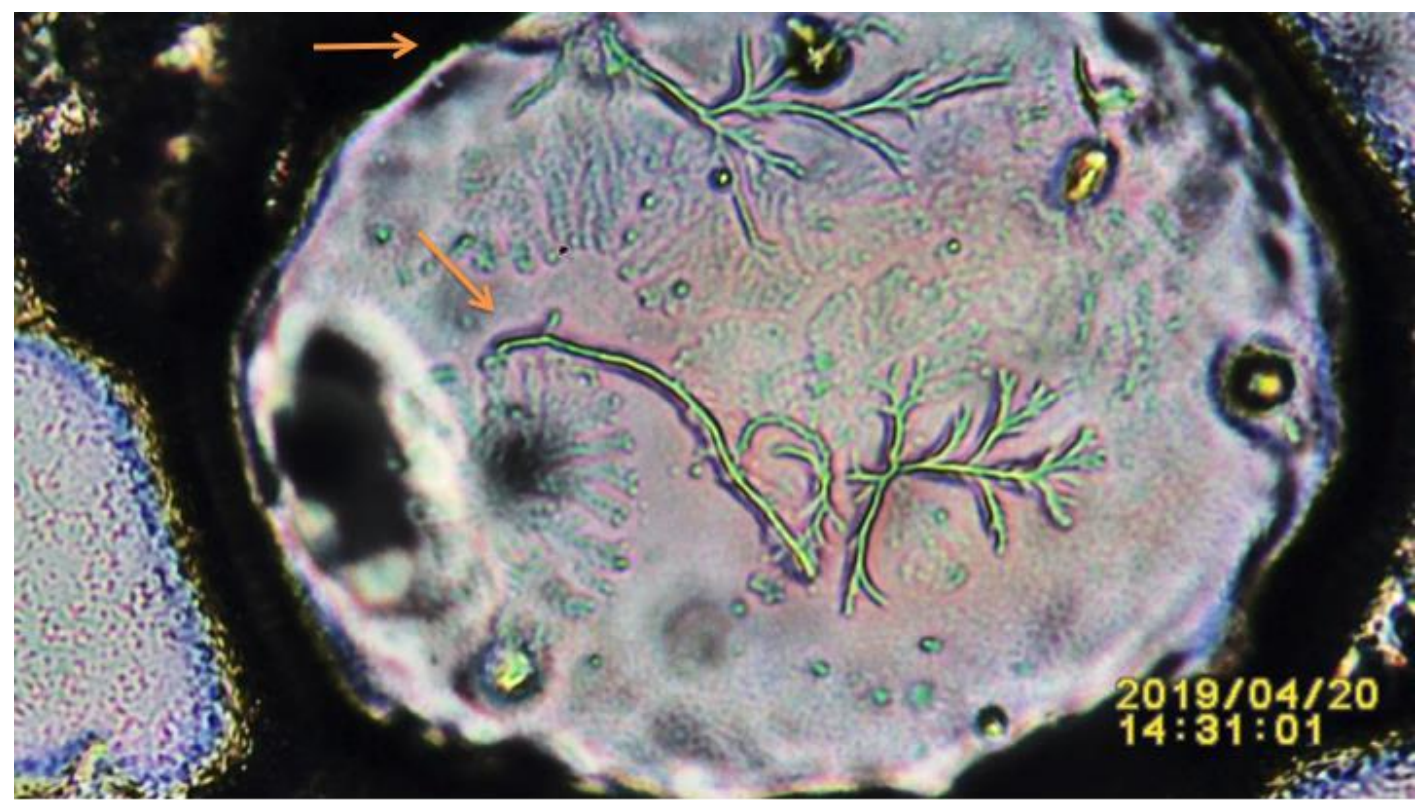

Figure 7: Orange Arrows= Amplified electrical discharges (shown in Figure 6) caused by bursting $\mathrm{O} 2$ bubbles penetrating a $1 \mathrm{~mm}$ glass barrier. Image as seen through an air bubble acting as a magnifying lens.

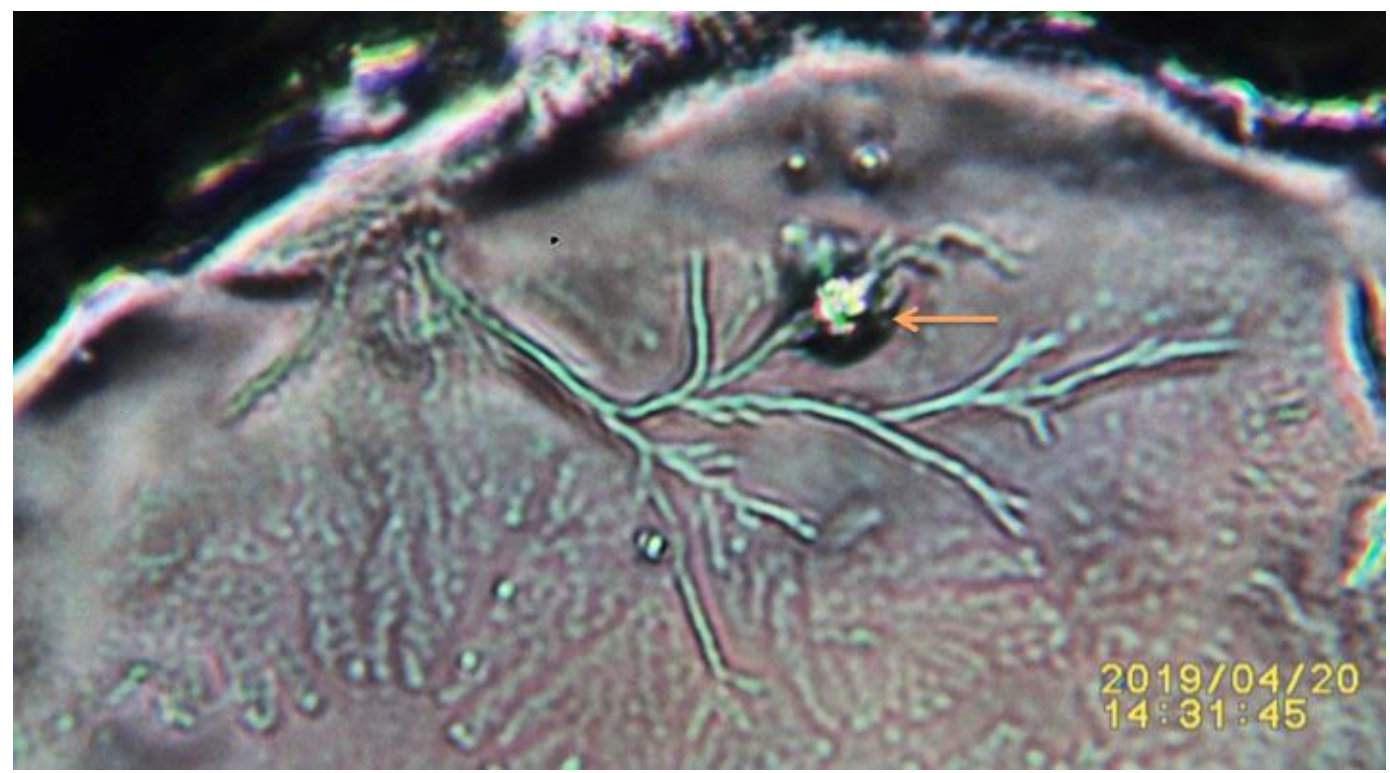

Figure 8: Static electricity discharge from saliva crystal illuminating unidentified material in its path.

\section{Medical Implications}

\section{Combined New Paradigm in the Cancer War}

\section{The Merging of Molecular and Biophysics Treatments}

Based on the current knowledge and the data presented in this manuscript, treatments methods of merging biophysical and molecular modalities are recommended. This is justified, since there is a possibility that the blocking of shockwave energy could aid in the cancer war. 


\section{Abbreviations, Terms:}

DNA= Deoxyribonucleic acid, a self-replicating material which is present in nearly all living organisms as the main constituent of chromosomes;

REGENERATIVE SUBSTANCE= Examples are lipids on leaves that regenerate within a few hours.

ROS= Reactive Oxygen Species;

$\mathrm{SDW}=$ When a substance is placed between two equally sized glass slides; $\mathrm{SSP}=$ When material is placed on slide surface.

\section{References}

[1] Harman D. Aging: a theory based on free radical and radiation chemistry. J Gerontol. 1956;11(3):298-300. doi: 10.1093/geronj/11.3.298.

[2] Embi A. A. Endogenous electromagnetic forces emissions during cell respiration as additional factor in cancer origin. Cancer cell international, 2016. 16, 60. doi:10.1186/s12935-016-0337-y16: 60. Published online 2016 Jul 28. doi: 10.1186/s12935-016-0337-y PMID: 27471435

[3] S Baig et al. (2016) Potential of apoptotic pathway-targeted cancer therapeutic research: Where do we stand? Cell Death \& Disease 2016 volume 7, page e2058

[4] Megan L. Sulciner et al. (2018) Targeting lipid mediators in cancer biology. Cancer and Metastasis Reviews September 2018, Volume 37, Issue 2-3, pp 557-572.

[5] Walther TC, Chung J, Farese RV Jr. (2017) Lipid Droplet Biogenesis. Annu Rev Cell Dev Biol. 2017 Oct 6;33:491-510. doi: 10.1146/annurev-cellbio-100616-060608.

[6] Dong Wook Kim Sang-Woo Kim Unyong Jeong. (2018) Lipids: Source of Static Electricity of Regenerative Natural Substances and Nondestructive Energy Harvesting. Advance materials. https://doi.org/10.1002/adma.201804949

[7] Embi AA, (2019) "THE PARAMAGNETISM AND SHOCK WAVES EFFECTS OF INTACT OR RUPTURED OXYGEN BUBBLES CAUSING PROKARYOTIC AND EUKARYOTIC CELLS DEFORMATIONS: SUPPORTING THE HYPOTHESIS OF CELL RESPIRATION AS ADDITIONAL FACTOR IN CANCER ORIGING." International Journal of Research Granthaalayah, 2019 7(3), 177-188. https://doi.org/10.5281/zenodo.2619530.

[8] DNA EXTRACTION- Michigan DNA DAY. Instructions https://documentcloud.adobe.com/link/track?uri=urn\%3Aaaid\%3Ascds\%3AUS\%3A194b92b9a820-4e14-95f0-a067e13a966f

[9] Liu Q et al. Targeting lipid metabolism of cancer cells: A promising therapeutic strategy for cancer. (2018) Cancer Lett. Cancer Metastasis Rev. 2018 Sep;37(2-3):557-572. doi: 10.1007/s10555-0189754-9.

[10] Sulciner, M.L., Gartung, A., Gilligan, M.M., Serhan, C.N., \& Panigrahy, D. Targeting lipid mediators in cancer biology.(2018) Cancer and Metastasis Reviews 2018, 37, 557-572.

[11] Ratushny, V Gober, M. D., Hick, R., Ridky, T. W., \& Seykora, J. T. (2012) From keratinocyte to cancer: the pathogenesis and modeling of cutaneous squamous cell carcinoma. The Journal of clinical investigation, 2012 122(2), 464-72.

[12] Na, N., Chandel, N. S., Litvan, J., \& Ridge, K. M. (2010) Mitochondrial reactive oxygen species are required for hypoxia-induced degradation of keratin intermediate filaments. FASEB journal 2010: official publication of the Federation of American Societies for Experimental Biology, 24.

[13] Ponec M, Weerheim A, Kempenaar J, et al. (1988) Lipid composition of cultured human keratinocytes in relation to their differentiation. J Lipid Res. 1988 Jul;29(7):949-61

[14] Jacobson, J., \& Sherlag, B. (2015). Aging and magnetism: Presenting a possible new holistic paradigm for ameliorating the aging process and the effects thereof, through externally applied physiologic PicoTesla magnetic fields. Medical hypotheses, 85 3, 276-86. 
[15] Jacobson JI (2016) Analysis: Magnetic Resonance Targets Telomeres/Telomerase for Cancer Treatment? Innov Ener Res 5: 135.

[16] Dong Wook Kim Sang-Woo Kim Unyong Jeong. (2018) Lipids: Source of Static Electricity of Regenerative Natural Substances and Nondestructive Energy Harvesting. Advance materials. https://doi.org/10.1002/adma.201804949

[17] Zhang C, Yang L. Ding Y. et al. Bacterial lipid droplets bind to DNA via an intermediary protein that enhances survival under stress. (2017) Nat Commun. Jul 6;8:15979. doi: 10.1038/ncomms 15979 .

*Corresponding author.

E-mail address: embi21@ att.net 